\title{
OTIMIZAÇÃO TOPOLÓGICA 3D SOB RESTRIÇÃO DE TENSÃO
}

\author{
Karilany Dantas Coutinho \\ Universidade Federal do Rio Grande do Norte, Departamento de Engenharia \\ Biomédica, Campus Universitário, Lagoa Nova, Natal, RN, Brasil. CEP: 59072-970. \\ karilany@ufrnet.br \\ João Carlos Arantes Costa Júnior \\ Universidade Federal do Rio Grande do Norte, Departamento de Engenharia Mecânica, \\ Campus Universitário, Lagoa Nova, Natal, RN, Brasil. CEP: 59072-970, Fax: (84) \\ 3215-3768, Fone: (84) 32153740, r-207. arantes@ ufnet.br \\ Marcelo Krajnc Alves \\ Universidade Federal de Santa Catarina, Departamento de Engenharia Mecânica, C.P. \\ 476, CEP 88010-970, Florianópolis, SC, Brasil. Fax: (48) 2343131, Fone: (48) \\ 3319899. krajnc@emc.ufsc.br \\ Custódio Leopoldino Brito Guerra Neto \\ Universidade Federal do Rio Grande do Norte, Departamento de Engenharia \\ Biomédica, Campus Universitário, Lagoa Nova, Natal, RN, Brasil. CEP: 59072-970. \\ custodioguerra@yahoo.com.br \\ Caroline Dantas Vilar Wanderley \\ Universidade Federal do Rio Grande do Norte, Departamento de Engenharia \\ Biomédica, Campus Universitário, Lagoa Nova, Natal, RN, Brasil. CEP: 59072-970. \\ carolinevilar@gmail.com
}

\section{RESUMO}

O problema de otimização topológica é utilizado para caracterizar e determinar a distribuição ótima de material no espaço de projeto. Em outras palavras, após serem definidas as condições de contorno num domínio de projeto pré-estabelecido, o problema é como distribuir o material de modo a solucionar o problema de minimização. Sendo assim, o problema de otimização de leiaute pode ser considerado como um problema pontual material/vazio. Este trabalho tem como objetivo propor um processo metodológico para a determinação de leiautes estruturais ótimos, que seja competitivo para utilização em problemas $3 \mathrm{D}$, minimizando a massa e satisfazendo um critério de tensão. O método de otimização de leiaute empregado é baseado na abordagem material, que considera uma equação constitutiva homogeneizada dependente apenas da densidade relativa do material. Para a descrição das propriedades do material, utiliza-se uma formulação de microestruturas porosas, caracterizado pelo modelo material do tipo SIMP. Na aproximação por elementos finitos utiliza-se um elemento tetraedro de quatro nós, que interpola linearmente o campo de densidades relativas além das componentes do campo de deslocamentos. Com as considerações propostas foi obtido um leiaute que caracteriza a topologia estrutural de problemas 3D, atendendo a solução de problema de modo competitivo. A formulação se mostrou promissora para a implementação de recursos de adaptatividade.

\section{PALAVRAS-CHAVE:}

topologia, leiaute, otimização, tensão, MEF. 


\title{
3D TOPOLOGY OPTIMIZATION WITH STRESS CONSTRAINT
}

\begin{abstract}
The Topology Optimization Problem consists in the total mass minimization of a structure. In order to solve three-dimensional stress problem, the Galerkin Finite Element Method was applied. It considers a four nodes tetrahedron finite element which interpolates not only the displacement fields, but also the relative density field. This work proposes to combine a stabilized element to avoid checkerboard problems, with a selective integration scheme to avoid volumetric locking. The optimal objective function is to minimize the mass, subjected to: a stress criterion (failed function); side and stability constraints to avoid checkerboard instability problems. Moreover, the design variables are given by the nodal relative densities of the finite element mesh using the SIMP material. With the proposed considerations it was obtained a layout that characterizes the structural topology of problems 3D, assisting the solution of problem in a competitive way. The formulation was shown promising for the implementation of adaptivity resources.
\end{abstract}

KEYWORDS:

topology, layout, optimization, minimization, FEM.

\section{OTIMIZAÇÃO TOPOLÓGICA 3D SOB RESTRIÇÃO DE TENSÃO}

\section{INTRODUÇÃO}

O objetivo deste trabalho é desenvolver um processo computacional que determine a topologia ótima de estruturas e componentes submetidos a carregamento mecânicos, Bendsoe \& Kikuchi (1988). A formulação do problema de minimização é desenvolvida para modelos tridimensionais. Para amenizar o custo computacional foi utilizado um clássico elemento finito de interpolação linear: tetraedro de quatro nós, com esquema de integração seletiva para contornar o efeito de travamento volumétrico. O problema de ótimo visa minimizar a massa satisfazendo um critério de tensão. Uma representação esquemática do problema de otimização topológica pode ser verificada na Fig. (1).

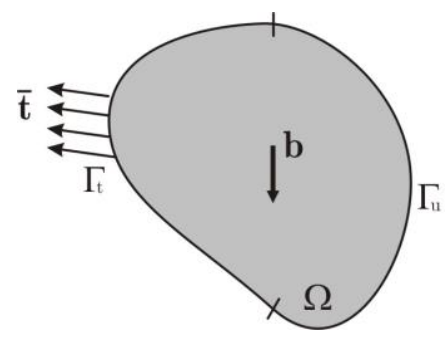

(a) domínio inicial

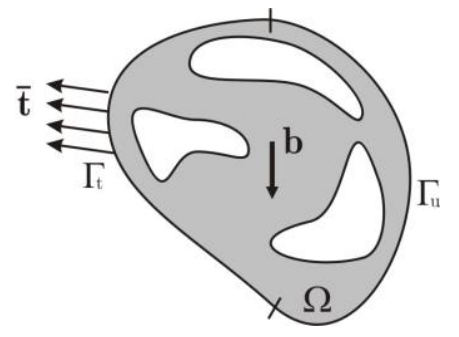

(b) domínio final

Sendo,

Figura 1- Caracterização do problema de otimização de leiaute.

- $\Omega$ o domínio do corpo a ser considerado;

- $\partial \Omega$ o contorno do domínio, tal que, $\partial \Omega=\Gamma_{\mathrm{u}} \cup \Gamma_{\mathrm{t}}$ e $\Gamma_{\mathrm{u}} \cap \Gamma_{\mathrm{t}}=\varnothing$; 
- $\quad \Gamma_{\mathrm{u}}$ a parte do contorno com deslocamento prescrito, i.e., $\mathbf{u}=\overline{\mathbf{u}}$;

- $\quad \Gamma_{\mathrm{t}}$ a parte do contorno com tração prescrita, i.e., $\mathbf{t}=\overline{\mathbf{t}}$;

- $\quad$ b a força de corpo.

\section{DEFINIÇÃO DO PROBLEMA}

\section{Formulação do Problema}

O campo de deslocamento $\mathbf{u}(\rho(\mathbf{x}), \mathbf{x})$ é a solução de:

$$
a(\mathbf{u}, \mathbf{v})=l(\mathbf{v}), \quad \forall \mathbf{v} \in H_{\mathrm{o}}
$$

sendo

$$
a(\mathbf{u}, \mathbf{v})=\int_{\Omega} \mathbf{D}^{H}(\rho) \boldsymbol{\varepsilon}(\mathbf{u}) \cdot \boldsymbol{\varepsilon}(\mathbf{v}) d \Omega
$$

Sendo a integral definida na equação (2) aproximada por uma integração seletiva, de modo que:

$$
a(\mathbf{u}, \mathrm{v})=a_{v o l}(\mathbf{u}, \mathrm{v})+a_{d i s t}(\mathbf{u}, \mathrm{v})
$$

onde, $a_{\text {vol }}(\mathbf{u}, \mathbf{v})$ são os termos volumétricos e $a_{\text {dist }}(\mathbf{u}, \mathbf{v})$ são os termos de distorção.

$\mathrm{e}$

$$
l \mathbf{v}=\int_{\Omega} \mathbf{b} \cdot \mathbf{v} d \Omega+\int_{\Gamma_{\mathbf{t}}} \mathbf{t} \cdot \mathbf{v} d \Gamma
$$

$H_{\mathrm{o}}(\Omega)=\mathbf{v} \mid \mathbf{v} \in\left[\begin{array}{ll}H^{1} & \Omega\end{array}\right]^{2}, \mathbf{v}=\mathbf{0}$ em $\mathbf{x} \in \Gamma_{\mathbf{u}} \quad$ e $H=\overline{\mathbf{u}}+H_{\mathrm{o}} \quad$ são os conjuntos das variações e deslocamentos admissíveis respectivamente.

\section{Definição do Modelo Material}

Para formular o problema de otimização de leiaute, aplica-se uma técnica de relaxação do problema que consiste na utilização de um modelo de material artificial caracterizado pela presença de microestruturas porosas do tipo SIMP (Solid Isotropic Material with Penalty), Bendsoe (1995) e Bendsoe \& Sigmund (1999), parametrizadas por sua densidade relativa.

$$
E \rho=\rho^{\eta} E_{\mathrm{o}} \text {. }
$$

Aqui, $E_{\mathrm{o}}$ representa o módulo de Young do material sólido e $\eta$ denota o parâmetro de penalização.

A equação constitutiva homogeneizada pode ser definida como:

$$
\boldsymbol{\sigma}=\left[\begin{array}{ll}
\mathbf{D}^{H} & \rho
\end{array}\right] \boldsymbol{\varepsilon}
$$

no qual $\sigma^{\mathrm{T}}=\sigma_{x x}, \sigma_{y y}, \sigma_{z z}, \sigma_{x y}, \sigma_{y z}, \sigma_{z x}$.

Aplicando o esquema de integração seletiva, teremos que:

$$
\left[\mathbf{D}^{H}(\rho)\right]=\left[\mathbf{D}_{v o l}^{H}\right]+\left[\mathbf{D}_{\text {dist }}^{H}\right] \text {. }
$$


Sub-integrando os termos volumétricos:

$$
\left[\mathbf{D}_{v o l}^{H}\right]=\left[\begin{array}{cc}
\mathbf{D}_{11}^{H}(\rho) & 0 \\
0 & 0
\end{array}\right], \varepsilon^{\mathrm{T}}=\left\{\varepsilon_{x x}, \varepsilon_{y y}, \varepsilon_{z z}, 0,0,0\right\}
$$

e integrando os termos de distorção,

$$
\left[\mathbf{D}_{\text {dist }}^{H}\right]=\left[\begin{array}{cc}
0 & 0 \\
0 & \mathbf{D}_{22}^{H}(\rho)
\end{array}\right], \quad \varepsilon^{\mathrm{T}}=\left\{0,0,0, \gamma_{x y}, \gamma_{y z}, \gamma_{z x}\right\}
$$

com

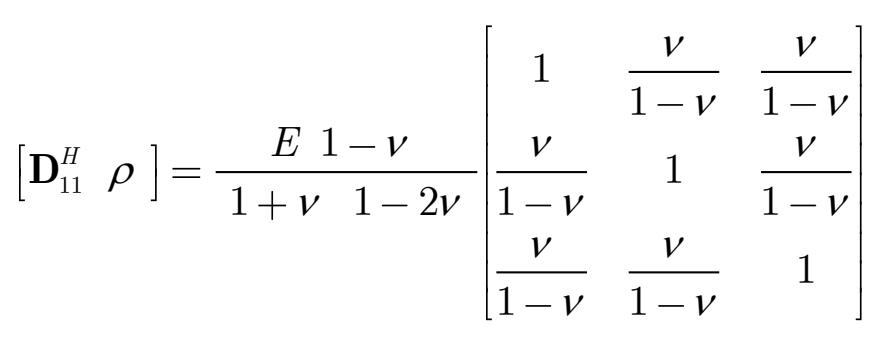

$$
\begin{aligned}
& {\left[\begin{array}{ll}
\mathbf{D}_{22}^{H} & \rho
\end{array}\right]=\frac{E}{21+v}\left[\begin{array}{lll}
1 & 0 & 0 \\
0 & 1 & 0 \\
0 & 0 & 1
\end{array}\right]}
\end{aligned}
$$

sendo $v$ o coeficiente de Poisson.

Além disso, com o objetivo de considerar restrições de tensão, é definida uma medida para a tensão efetiva $\sigma^{*}(\rho)$, de acordo com os trabalhos de Duysinx \& Sigmund (1998) e Duysinx \& Bendsoe (1998), dada por:

$$
\sigma^{*} \rho=\frac{\sigma \rho}{\rho^{\eta}}
$$

Agora, com a tensão efetiva definida pela eq.(12), introduz-se uma restrição de tensão local, que define um critério de falha do material em termos da tensão efetiva de von Mises, dada como:

$$
\sigma_{e q}^{*} \rho=\frac{\sigma_{e q} \rho}{\rho^{\eta}} \leq \sigma_{y}
$$

onde $\sigma_{y}$ é a tensão admissível.

\section{Critério de Tensão de Falha com Relaxação}

A formulação do problema de otimização topológica de um corpo tridimensional, sujeito à restrições de tensão com relaxação $\kappa$, consiste na determinação do campo de densidade relativa $\rho \mathbf{x}$. O problema de otimização topológica sob restrições de tensão local está sujeito a problemas de singularidade, devido à degeneração do espaço de projeto. Para contornar o problema de singularidade, como proposto por Cheng \& Guo (1997), considera-se uma relaxação $\kappa$ na restrição de tensão, de modo que 


$$
\rho\left(\frac{\sigma_{e q}^{*}}{\sigma_{y}}-1\right)+\rho \kappa \rho_{\text {sup }}-\rho \leq 0 .
$$

Esta relaxação atua sobre a parte degenerada do espaço de projeto, possibilitando a criação e remoção de furos sem violar a restrição de tensão.

Desta forma, o problema pode ser formulado como:

$$
\min \int_{\Omega} \rho \mathbf{x} d \Omega
$$

Tal que

$$
\begin{aligned}
& \rho\left(\frac{\sigma_{e q}^{*}}{\sigma_{y}}-1\right)+\rho \kappa \quad \rho_{\text {sup }}-\rho \leq 0 \\
& \rho_{\text {inf }}-\rho \mathbf{x} \leq 0, \quad \rho \mathbf{x}-\rho_{\text {sup }} \leq 0 ; \\
& \left(\frac{\partial \rho \mathbf{x}}{\partial j}\right)^{2} \leq c_{j}^{2}, \quad p / j=x, y \text { e } z . \quad \forall \mathbf{x} \in \Omega
\end{aligned}
$$

\section{APROXIMAÇÃO DO PROBLEMA}

\section{Formulação Discreta do Problema}

O método numérico utilizado para aproximar o modelo estrutural é o Método dos Elementos Finitos de Galerkin. O elemento finito utilizado na aproximação é o tetraedro de quatro nós, que interpola o campo de densidades relativas $\rho$ além das componentes do campo de deslocamentos $u_{x}, u_{y}, u_{z}$, mostrado na Fig. (2).

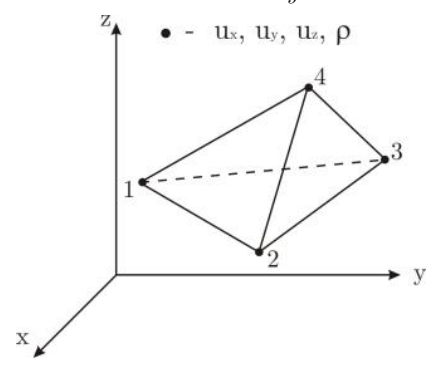

Figura 2- Campo de deslocamento e densidade.

As variáveis de projeto são definidas pela densidade relativa dos nós da malha. É importante notar que vários problemas de instabilidade de tabuleiro têm sido observados com a utilização de elementos de baixa ordem de interpolação. Entretanto, a imposição de uma restrição de limite sobre as componentes de $\nabla \rho$ tem mostrado ser muito eficiente, evitando o aparecimento destas instabilidades, Petersson \& Sigmund (1998) e Sigmund \& Petersson (1998).

O problema de otimização topológica pode ser aproximado, de forma discreta, do seguinte modo:

$$
\min \int_{\Omega} \rho \mathbf{x} d \Omega
$$


sujeito as restrições:

(i) Restrições de Tensão:

$$
\rho \mathbf{x}\left(\frac{\sigma_{e q}^{*} \rho \mathbf{x}, \mathbf{u} \rho \mathbf{x}, \mathbf{x}}{\sigma_{y}}-1\right)+\rho \mathbf{x} \kappa \rho_{\text {sup }}-\rho \mathbf{x} \leq 0
$$

A restrição de tensão efetiva com relaxação $\kappa$, definida pela eq. (18), deve ser satisfeita para todo $\mathbf{x} \in \Omega$, o que representa uma restrição paramétrica. Um método efetivo de trabalhar com restrições paramétricas consiste na relaxação da condição pontual (critério local) através da consideração de uma restrição integrada, isto é, da utilização de um critério global, Kohn \& Strang (1986). Neste trabalho, propõe-se o seguinte critério global:

$$
\bar{g} \boldsymbol{\rho}, \mathbf{u} \boldsymbol{\rho}=\left\{\frac{1}{\Omega} \int_{\Omega}\left\langle\rho\left(\frac{\sigma_{e q}^{*}}{\sigma_{y}}-1\right)+\rho \kappa \rho_{\text {sup }}-\rho\right\rangle^{p} d \Omega\right\}^{1 / p} \leq 0
$$

onde é definido que $\langle f \mathbf{x}\rangle=\max 0, f \mathbf{x}$, representando a parte positiva de $f \mathbf{x}$.

Esta medida global é denominada como média- $p$ integral. No caso de $p \rightarrow \infty$, a restrição média- $p$ aproxima, no limite, a restrição pontual

$$
\max _{\mathbf{x} \in \Omega}\left|\left\langle\rho\left(\frac{\sigma_{e q}^{*} \rho}{\sigma_{y}}-1\right)-\kappa 1-\rho\right\rangle\right| \leq 0 .
$$

Entretanto, quando $p \rightarrow \infty$ o problema torna-se mal condicionado, fazendo com que o critério de falha integral tenda a ser influenciado por apenas um valor de pico do campo de tensão, enquanto as outras restrições são ignoradas. Isto pode ocasionar um comportamento oscilatório do algoritmo de otimização, possibilitando a falha da convergência do processo de otimização.

(ii) Restrições Laterais

$$
\rho_{\text {inf }}-\rho \mathbf{x} \leq 0 \quad \text { e } \quad \rho \mathbf{x}-\rho_{\text {sup }} \leq 0, \quad \forall \mathbf{x} \in \Omega
$$

(iii) Restrições de Estabilidade

$$
\bar{h}_{j} \boldsymbol{\rho}=\left\{\frac{1}{\Omega} \int_{\Omega}\left\langle h_{e j} x_{e}\right\rangle^{p} d \Omega\right\}^{1 / /}, \quad \mathrm{p} / j=x, y \text { e } z
$$

sendo

$$
h_{e j} \rho=\frac{\sqrt{\left(\frac{\partial \rho}{\partial j}\right)^{2}}}{c_{j}}-1 \quad, \quad \mathrm{p} / j=x, y \text { e } z
$$

As constantes $c_{x}, c_{y}$ e $c_{z}$ impõem um limite superior às componentes do gradiente da densidade relativa. 
Considere um elemento genérico como mostrado na Fig.3, sendo $\mathbf{x}_{n}=x_{i}, y_{i}, z_{i}$, $\mathrm{p} / i=1, \ldots, 4$ as coordenadas dos vértices e $\mathbf{x}_{m}=x_{m}, y_{m}, z_{m}$ as coordenadas do baricentro do elemento tetraedro de quatro nós.

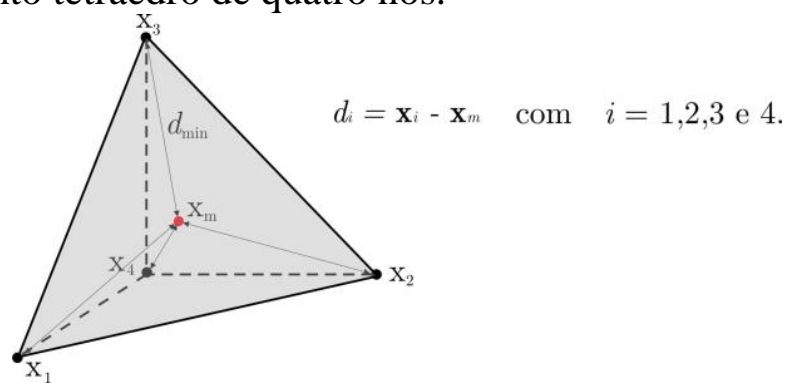

Figura 3- Coordenadas do elemento finito tetraedro de quatro nós.

denotando:

$$
d_{\min }=\min _{j}\left\|\mathbf{d}_{j}\right\| \quad \mathrm{p} / \quad i=1, \ldots, 4
$$

sendo adotado que

$$
c_{x}^{e}=c_{y}^{e}=c_{z}^{e}=\frac{1}{d_{\min }} .
$$

Para $\boldsymbol{\rho} \in \mathbf{X} \operatorname{com} \mathbf{X}=\boldsymbol{\rho} \in \mathbb{R}^{n} \mid \rho_{n}^{\mathrm{inf}} \leq \rho_{n} \leq \rho_{n}^{\text {sup }}$

Para resolver o problema de otimização é aplicado o Método do Lagrangeano Aumentado de maneira a obter um problema de ótimo apenas com restrições laterais. $\mathrm{O}$ processo de otimização agora consiste na solução de uma seqüência de problemas de mínimo com restrições do tipo caixa, o qual pode ser resumido como:

1. Definir valores iniciais: $k=0, \lambda^{k}=0, \mu^{\mathrm{k}}=\mathbf{0}$, erro $=1,0, \zeta$, $\boldsymbol{\omega}^{k}$ e tol.

2. Enquanto erro $>$ tol, efetuar laço:

(i) Solução do problema de minimização com restrições laterais

$$
\min \Pi \rho, \lambda, \mu ; \zeta, \omega, \quad \forall \rho \in \mathbf{X}
$$

onde,

$$
\Pi \boldsymbol{\rho}, \lambda, \boldsymbol{\mu} ; \zeta, \boldsymbol{\omega}=f \boldsymbol{\rho}+\frac{1}{\zeta} \sum_{e=1}^{n_{e}} \Lambda_{e} g_{e}, \zeta \lambda_{e}+\sum_{j=1}^{3}\left[\frac{1}{\omega_{j}} \sum_{e=1}^{n_{e}} \Psi_{e}^{j} h_{e}^{j}, \omega_{j} \mu_{e j}\right]
$$

com

$$
\Lambda_{e} g_{e}, \zeta \lambda_{e}=\left\{\begin{array}{cc}
g_{e} g_{e}+\zeta \lambda_{e} & , \quad \text { se } g_{e} \geq-\frac{\zeta \lambda_{e}}{2} \\
-\left(\frac{\zeta \lambda_{e}}{2}\right)^{2} & , \quad \text { se } g_{e}<-\frac{\zeta \lambda_{e}}{2}
\end{array}\right.
$$

$\mathrm{e}$ 


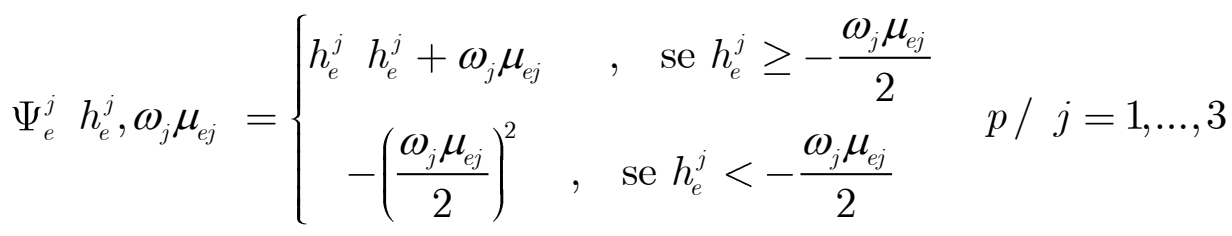

(ii) Atualização dos multiplicadores de Lagrange

$$
\lambda_{e}^{k+1}=\max \left\{0, \lambda_{e}^{k}+\frac{2}{\zeta} g_{e} \mathbf{x}^{k}\right\}
$$

$\mathrm{e}$

$$
\mu_{e j}^{k+1}=\max \left\{0, \mu_{e j}^{k}+\frac{2}{\omega_{j}} h_{e} \mathbf{x}^{k}\right\} \quad p / j=1, \ldots, 3 .
$$

(iii) Atualização dos parâmetros de penalidade

$$
\zeta^{k+1}=\left\{\begin{array}{cc}
\gamma_{1} \zeta^{k} & , \text { se } \gamma_{1} \zeta^{k}>\zeta^{c r i t} \\
\zeta^{c r i t}
\end{array}\right.
$$

$\mathrm{e}$

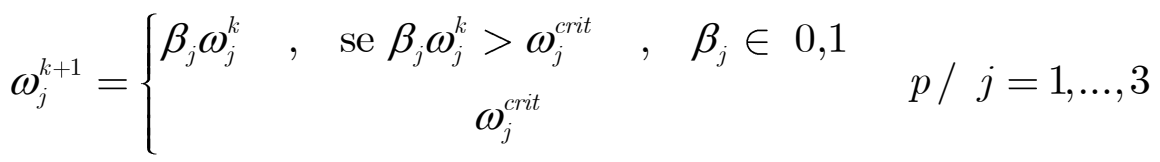

(iv) Determinação do erro

$a=\max _{e}\left|\lambda_{e}^{k+1}-\lambda_{e}\right|, b=\max _{e}\left|\mu_{e 1}^{k+1}-\mu_{e 1}\right|, c=\max _{e}\left|\mu_{e 2}^{k+1}-\mu_{e 2}\right|$ e $d=\max _{e}\left|\mu_{e 3}^{k+1}-\mu_{e 3}\right|$

então, erro $=\max a, b, c, d$.

3. Fim do laço.

Desta forma, o problema pode ser formulado como: Dado $\lambda, \mu_{1}, \mu_{2}, \mu_{3} \in \mathbb{R}^{p} \mathrm{e}$ $\zeta, \omega_{1}, \omega_{2}, \omega_{3} \in \mathbb{R}$. O problema consiste em encontrar um $\rho^{*} \in \mathbb{R}^{n}$ tal que:

$$
\boldsymbol{\rho}^{*}=\arg \min \Pi \boldsymbol{\rho}, \lambda, \boldsymbol{\mu} ; \zeta, \boldsymbol{\omega} \quad, \quad \forall \boldsymbol{\rho} \in \mathbf{X} .
$$

\section{PROBLEMAS}

\section{Problema 01}

Aqui nós consideramos o problema ilustrado na figura na Fig. (4). No qual as propriedades do material são dadas por: módulo de Young, $E=215 \times 10^{9} \mathrm{~N} / \mathrm{m}^{2}$; coeficiente de Poisson, $v=0,3$; limite de resistência; $S_{y}=260 \times 10^{6} \mathrm{~N} / \mathrm{m}^{2}$; carga Aplicada, $\mathbf{W}=150 \times 10^{6} \mathrm{~N}$; dimensões, $a=5 \mathrm{~m}, b=20 \mathrm{~m}$ e $c=4 \mathrm{~m}$. 
Para simplificar o problema, analisou-se 1/4 de simetria da estrutura com 11133 elementos e 2337 nós.
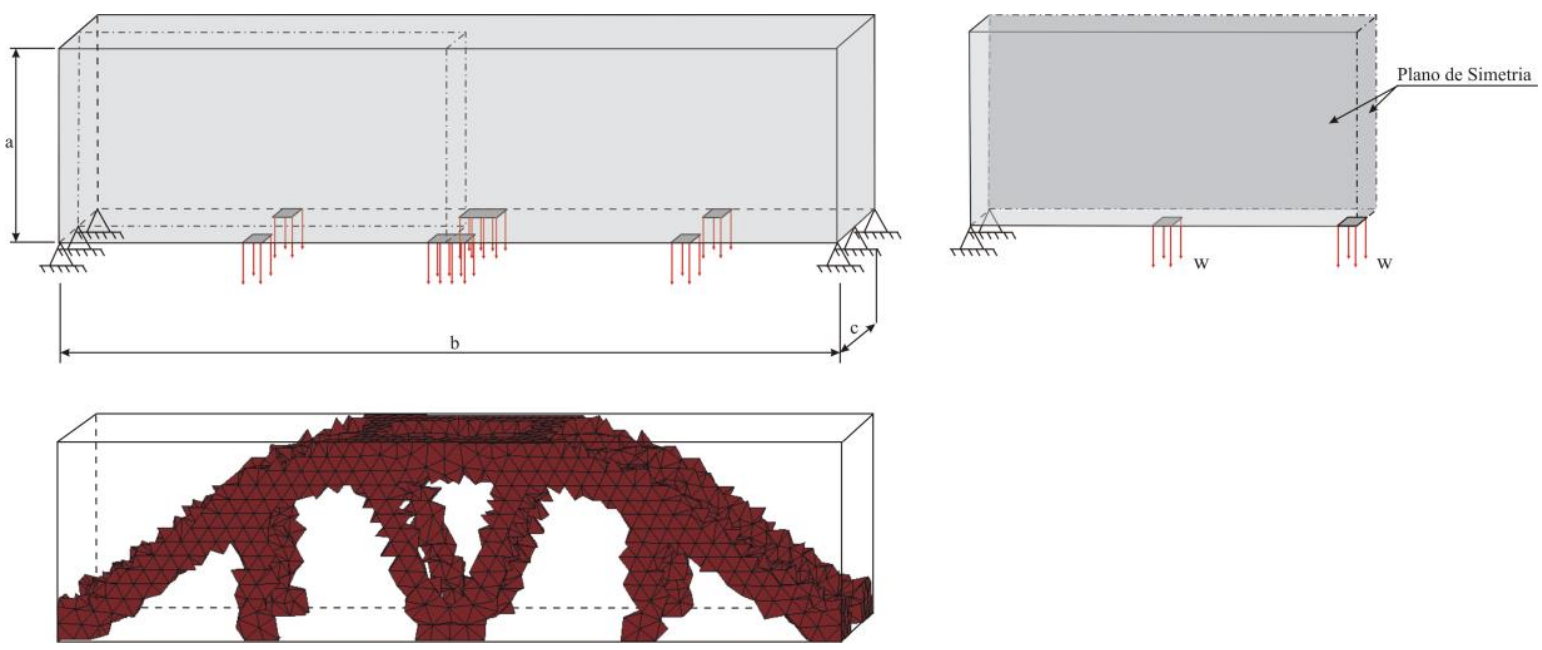

Figura 4- Resultado do Problema 1.

\section{Problema 02}

Aqui o problema consiste em um bloco cujo as dimensões são $a=4 m, b=6 m$ e $c=2 m$, no qual é submetida uma carga de $\mathbf{W}=150 \times 10^{6} \mathrm{~N}$, como ilustrado na Fig. (5). O bloco está fixo em $\bar{\Omega}$ nas três direções $x, y$ e $z$. Ou seja, $\bar{\Omega}_{1}=\bar{\Omega}_{2}=\bar{\Omega}_{3}=\bar{\Omega}_{4}$ com deslocamento prescrito $0,0,0$..

Propriedades do material: módulo de Young, $E_{\mathrm{o}}=215 \times 10^{9} \mathrm{~N} / \mathrm{m}^{2}$; coeficiente de Poisson, $v=0,3$; tensão de escoamento; $S_{y}=260 \times 10^{6} \mathrm{~N} / \mathrm{m}^{2}$.

Para simplificar o problema, analisou-se $1 / 4$ de simetria da estrutura com 10651 elementos e 2261 nós.
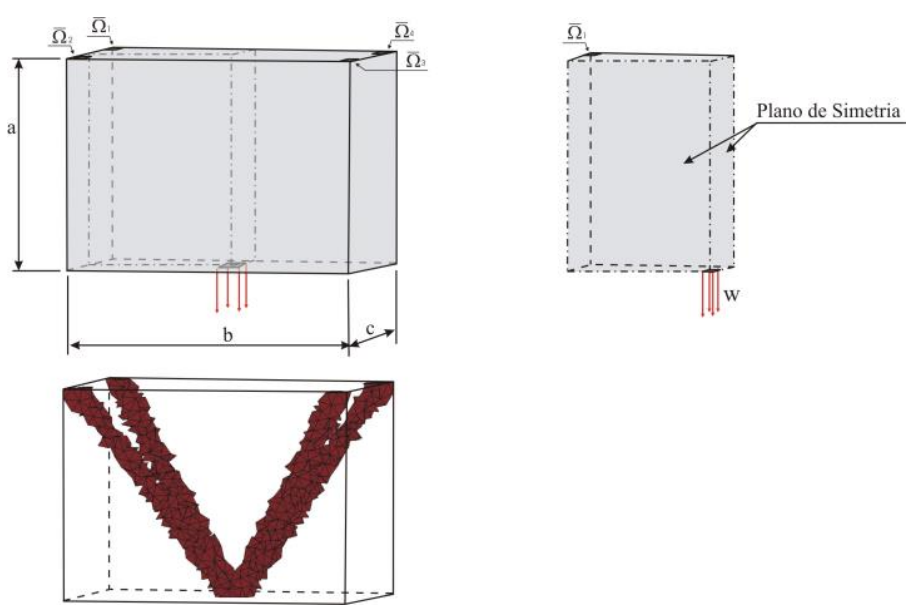

Figura 5- Resultado do Problema 2.

\section{CONCLUSÕES}

O método proposto mostrou ser efetivo e robusto na geração de um leiaute estrutural ótimo para problemas estruturais 3D. A resolução final do contorno material é 
diretamente dependente do tamanho médio do elemento finito empregado, representando a relação direta com o custo computacional. A formulação mostrou-se promissora para a implementação de recursos de adaptatividade, ou seja, a implementação de um processo inteligente de refinamento da malha com informações da topologia obtida na malha original, para que seja melhorada à definição do contorno da topologia com menor custo computacional, Costa Jr. (2003), Costa Jr. \& Alves (2003a-b). Para solucionar o problema de otimização não linear foi utilizado o código computacional da plataforma TANGO, Andreani et al (2004), Andreani et al (2005) e Birgin \& Martinez (2002).

\section{REFERÊNCIAS BIBLIOGRÁFICAS}

1. BENDSOE M. P.; Optimization of Structural Topology, Shape, and Material - , Berlin Heidelberg: Springer-Verlag, 1995, 271 p.

2. BENDSOE M. P., KIKUCHI N.; Generating optimal topologies in structural design using a homogenization method, Comput. Meth. Appl. Mech. Engrg., v. 71(2), p. 197-224, 1988

3. BENDSOE M. P., SIGMUND O.; Material interpolation schemes in topology optimization, Archive of Applied Mechanics, v. 69, p. 635-54, 1999.

4. CHENG, G. e GUO, X.; E-Relaxed Approach in Structural Topology Optimization, Structural Optimization, vol. 13, pp. 258-266, 1997.

5. COSTA JR. J. C. A.; Otimização Topológica com Refinos H-adaptativos, Tese de Doutorado, UFSC, Santa Catarina, Brasil, 2003.

6. COSTA JR. J. C. A., ALVES M. K.; h-adaptivity procedures of a stress based layout optimization, Cilamce 2003, Ouro Preto-MG, Brasil, CDRom media, 2003 b.

7. COSTA JR. J. C. A., ALVES M. K.; Layout optimization with h-adaptivity of structures, Int. J. Numer. Meth. Engng., v. 58(1), p. 83-102, $2003 \mathrm{a}$.

8. DUYSINX P., SIGMUND O.; New Development in handling stress constraints in optimal material distribution, In 7th AIAA/USAF/NASA/ISSMO Symposium on Multidisciplinary Design Optimization, American Institute of Aeronautics and Astronautics, Saint Louis, Missouri, EUA, paper 98/4906/1-9, 1998.

9. DUYSINX, P. e BENDSOE M. P.; Topology Optimization of Continuum Structures with Local Stress Constraints, Int. J. Numer. Meth. Engng., vol. 43, pp. 1453-1478, 1998. 
10. E. G. BIRGIN and J. M. MARTÍNEZ; Large-scale active-set box-constrained optimization method with spectral projected gradients, Computational Optimization and Applications 23, pp. 101-125, 2002.

11. KOHN R. V., STRANG. G.; Optimal design and relaxation of variational problem, Comm. Pure. Appl. Math., 39, p. 1-25, 139-82, 353-77, 1986.

12. PETERSSON J.; Sigmund O. Slope constrained topology optimization, Int. J. Numer. Meth. Engng., v. 41(8), 1417-34, 1998.

13. R. ANDREANI, E. G. BIRGIN, J. M. MARTÍNEZ and M. L. SCHUVERDT; Augmented Lagrangian methods under the Constant Positive Linear Dependence constraint qualification, Mathematical Programming, 2004.

14. R. ANDREANI, E. G. BIRGIN, J. M. MARTÍNEZ and M. L. SCHUVERDT; On Augmented Lagrangian methods with general lower-level constraints, Technical Report MCDO-050304, Department of Applied Mathematics, UNICAMP, Brazil, 2005.

15. SIGMUND O., PETERSSON J.; Numerical instabilities in topology optimization: a survey on procedures dealing with checkerboards, mesh dependencies and local minima, Structural Optimization, v. 16, p. 68-75, 1998. 